\title{
Are there MACHOs in the Milky Way halo?
}

\author{
A. M. Green ${ }^{1,2}$ and K. Jedamzik ${ }^{3,4}$ \\ ${ }^{1}$ Department of Physics, Stockholm University, 10691 Stockholm, Sweden ${ }^{\star}$ \\ e-mail: amg@physto.se \\ 2 Astronomy Unit, School of Mathematical Sciences, Queen Mary University of London, Mile End Road, London, E1 4NS, \\ UK \\ ${ }^{3}$ Laboratoire de Physique Mathématique et Théorique, Université de Montpellier II, 34095 Montpellier Cedex 5, France ${ }^{\star \star}$ \\ e-mail: jedamzik@lpm.univ-montp2.fr \\ ${ }^{4}$ Max-Planck-Institut für Astrophysik, 85740 Garching, Germany
}

Received 12 June 2002 / Accepted 5 September 2002

\begin{abstract}
Microlensing searches aim to detect compact halo dark matter via its gravitational lensing effect on stars within the Large Magellanic Cloud. These searches have led to the claim that roughly one fifth of the galactic halo dark matter may be in the form of compact, solar-mass objects. We analyze this hypothesis by considering the goodness-of-fit of the best-fit halo dark matter solutions to the observational data. We show that the distribution of the durations of the observed microlensing events is significantly narrower than that expected to result from a standard halo lens population at 90 to $95 \%$ confidence, casting doubt on the lenses constituting halo dark matter. This conclusion may possibly be avoided if (i) the Milky Way halo is sufficiently nonstandard or (ii) a large fraction of the events are due to non-halo populations with event durations coincidentally close to those of the putative halo population or (iii) individual event durations have been seriously underestimated due to blending.
\end{abstract}

Key words. Galaxy: halo - cosmology: dark matter

\section{Introduction}

Massive compact halo objects (MACHOs) with mass in the range $10^{-8} M_{\odot}$ to $10^{3} M_{\odot}$ can be detected via the temporary amplification of background stars which occurs, due to gravitational microlensing, when the MACHO passes close to the line of sight to a background star (Paczyński 1986). In the early 1990s several collaborations began monitoring millions of stars in the Large and Small Magellanic Clouds (LMC and SMC) ${ }^{1}$, in order to search for Milky Way (MW) compact halo dark matter, and indeed a number of candidate microlensing events have been observed (Alcock et al. 2000b; Lasserre et al. 2000; Milsztajn \& Lasserre 2001). The interpretation of these microlensing events is a matter of much debate. Though the lenses may easily reside within the MW halo and constitute halo dark matter, it is also possible that the contribution to the lensing rate due to other, so far unknown non-halo populations of objects has been underestimated (see e.g. Bennett 1998; Zhao 1999). In particular the lensing rate due to stars, or MACHOs, within the LMC itself (Wu 1994; Auborg et al. 1999; Salati et al. 1999;

\footnotetext{
Send offprint requests to: A. M. Green, e-mail: amg@physto. se

* Present address.

$\star \star$ Present address.

${ }^{1}$ http://wwwmacho.mcmaster.ca/, http://eros.in2p3.fr/
}

Evans \& Kerins 2000; Gyuk et al. 2000), or a very thick Milky Way disk (Gyuk \& Gates 2001) may be significant.

The EROS collaboration have five candidate events, resulting from three years of observation of 25 million stars in the LMC (Milsztajn \& Lasserre 2001). Due to the small number of events, and poor fit to microlensing of several of these events, they use these events to place constraints on the halo fraction as a function of lens mass, assuming a standard Maxwellian MW halo. In contrast, the MACHO collaboration claim that roughly $20 \%$ of the halo is in compact objects with $M \sim 0.5 M_{\odot}$, using their 13/17 candidates events which result from the 5.7 years of observation of 10.7 million stars (Alcock et al. 2000b). While not in direct conflict with the EROS exclusion limits these MACHO best fit parameters lie just outside the EROS exclusion limits. These differences could be due to EROS covering a larger solid angle than MACHO, such that the lensing rate due to lenses in the LMC itself should be smaller, as well as the use of less crowded fields by EROS as compared to MACHO, simplifying the estimation of the event durations. See Milsztajn (2002) for an extended discussion of the current observational situation.

In this paper we revisit the analysis of the MACHO collaborations candidate microlensing events (Alcock et al. 2000b). We contemplate whether the advocated interpretation of the microlensing observations in terms of a MACHO halo dark matter component with Maxwellian velocity distribution within 
a standard MW halo and with best-fit typical mass $0.6 M_{\odot}$ and halo dark matter fraction $f \approx 20 \%$ actually provides, in absolute terms, a good fit to the data.

\section{Candidate events}

The MACHO collaboration apply two sets of selection criteria to their data. Criteria $\mathrm{A}$ is the most restrictive, only accepting events with a single highly significant bump in their lightcurve as expected for simple, single lens, events, and results in 13 events. Criteria B is looser with 17 events passing the selection criteria, being designed to also accept low signal to noise events and exotic events, such as those where the lens is binary and the lightcurve has distinctive caustic features. Marginal events which are suspected of being supernovae occurring in host galaxies behind the LMC are rejected from criteria A but kept in criteria B. Further study of event number $22^{2}$ has found that the source is extended and has emission lines uncharacteristic of a stellar object and it is hence very unlikely a microlensing event (Alcock et al. 2001a). This extraordinarily long event will henceforth be excluded from the sample, leaving 16 candidate events for criteria B.

Known stellar populations within the MW disk and bulge, as well as within the LMC disk, are expected to account for approximately one quarter of the observed events (Alcock et al. 2000b). Follow-up observations of fields containing each of the source stars have been carried out using the Hubble Space Telescope Wide Field Planetary Camera 2 (Alcock et al. 2001b). In the case of event number 5 (which passes both sets of selection criteria) a faint red object has been detected close to the source star. A chance super-position is extremely unlikely and a combined analysis of the HST and microlensing data finds that this object is most likely an M dwarf located in the MW disk.

It is possible to obtain more information about the properties of a given lens if either the lens or source is within a binary, as the microlensing lightcurve then exhibits additional features. The lens responsible for event 9 is a binary, producing distinctive caustic features in the lightcurve from which the source crossing radius and hence the lens projected velocity could be estimated (Bennett et al. 1996; Alcock et al. 2000a). The low projected velocity found suggests that either the lens resides in the LMC disk or the source star is also binary. Accurate photometry of event number 14 (which passes both sets of selection criteria) revealed a periodic modulation in the lightcurve. Fits to a rotating binary source have been performed which indicate that it is most likely that the lens resides in the LMC disc, though direct spectral observations of the source are required to confirm this (Alcock et al. 2001c).

While there is clear evidence that event number 22 is not due to microlensing and that event 5 is due to a non MW halo lens, the locations of the lenses responsible for events 9 and 14 are not unambiguously known. Furthermore no conclusions on the locations of the entire lens population can be drawn from the locations of the lenses responsible for events 9 and 14,

\footnotetext{
2 We use the MACHO collaboration's event numbering nomenclature (Alcock et al. 2000b) throughout.
}

due to the biases involved in observing exotic events (Alcock et al. 2001c; Honma 1999).

A further complication is the estimation of the event durations. In crowded stellar fields it is not possible to resolve individual stars and some fraction of the baseline flux may come from unlensed stars. This phenomena, known as blending, results in an underestimate of the event timescale. The MACHO collaboration take this into account by carrying out sophisticated Monte Carlo simulations to find the average factor by which the real timescale is underestimated, and then correcting each of the fitted durations by this factor (Alcock et al. 2000b; Alcock et al. 2001a).

\section{Likelihood analysis}

The MACHO collaboration defines the likelihood of a given model as the product of the Poisson probability of observing $N_{\text {obs }}$ events when expecting $N_{\exp }$ events and the probabilities of finding the observed durations $\hat{t}_{\mathrm{j}}$ (where $j=1, \ldots, N_{\mathrm{obs}}$ ) from the theoretical duration distribution (Alcock et al. 1996; Alcock et al. 1997):

$\mathcal{L}=\exp \left(-N_{\text {exp }}\right) \Pi_{j=1}^{N_{\text {obs }}} \mu_{j}$,

where the expected number of events is given by

$N_{\text {exp }}=E \int_{0}^{\infty} \frac{\mathrm{d} \Gamma}{\mathrm{d} t} \epsilon(t) \mathrm{d} t$,

and $\mu_{j}$ by

$\mu_{j}=E \epsilon\left(t_{j}\right) \frac{\mathrm{d} \Gamma\left(t_{j}\right)}{\mathrm{d} t}$.

Here $E=6.12 \times 10^{7}$ star-years is the exposure, $\epsilon(t)$ is the detection efficiency, $t$ is the estimated event duration statistically corrected for blending and $\mathrm{d} \Gamma / \mathrm{d} t$ is the differential event rate. The likelihood approach can be used to compare how well different sets of parameter values within a given theoretical model fit the data. In the present context these parameters are the MACHO mass and halo fraction and the model is a standard halo population of compact dark matter. However, the likelihood approach does not tell one, in absolute terms, how good a fit really is. In particular, it is possible that though "best-fit" model parameters have been found, the model provides a generally bad description of the observational data. In order to illustrate this we compare $\mathrm{d} \Gamma / \mathrm{d} t$ of the best fit standard halo model with delta MACHO mass function (S-DMF) with that of a best fit (i.e. maximum likelihood) Gaussian differential event rate with variable amplitude, center and width. We note that the Gaussian has no physical motivation and is intended to simply provide some arbitrary reference to the S-DMF model ${ }^{3}$.

The best fit Gaussian and S-DMF differential event rates which arise in a likelihood analysis assuming that all 13 events found using criteria A are due to MW halo lenses (i.e. neglecting the event rate due to background events) are plotted in Fig. 1 multiplied by the detection efficiency. The Gaussian clearly gives a better fit to the observed event distribution than

\footnotetext{
${ }^{3}$ Note that the Gaussian model has one more fitting parameter than the S-DMF model.
} 


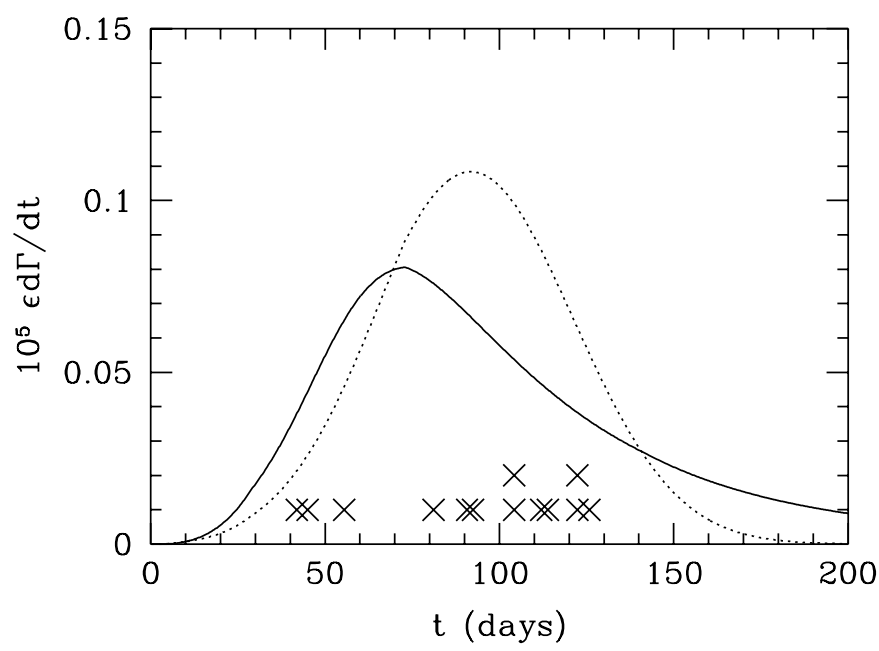

Fig. 1. The best fit delta function plus standard halo model (solid line) and Gaussian (dotted) differential event rates for the 13 events resulting from criteria A. Crosses indicate the observed event durations. An analogous plot for the 16 events resulting from criteria $B$ appears very similar.

the S-DMF model, and its likelihood is greater by a factor of 32. We have also carried out this comparison for all possible 10 event sub-sets (i.e. subtracted every possible combination of 3 background events) and in every case the Gaussian has a larger maximum likelihood (by a factor ranging from 5 to 4500 ).

\section{Event duration distribution}

Figure 1 illustrates the reason why the artificial Gaussian event duration distribution provides a better fit to the data than the event duration distribution produced by a MACHO population within a standard halo model; namely, whereas the S-DMF predicts the occurrence of long duration events none are observed. To quantify this discrepancy we have performed a Monte Carlo simulation which compares the observed width of the distribution

Width $=\frac{\operatorname{Max}_{\left\{j=1, N_{\mathrm{obs}}^{*}\right\}}\left(t_{j}\right)-\operatorname{Min}_{\left\{j=1, N_{\mathrm{obs}}^{*}\right\}}\left(t_{j}\right)}{\operatorname{Avg}_{\left\{j=1, N_{\mathrm{obs}}^{*}\right\}}\left(t_{j}\right)}$,

to the Width obtained by statistically drawing a number $N_{\mathrm{obs}}^{\text {halo }}$ of events from a standard halo event duration distribution (multiplied by the relevant detection efficiency and taking into account the transverse velocity of the line-of-sight). We note that the above statistic is designed to be independent of the best-fit MACHO halo fraction and $\operatorname{mass}^{4}$ and is thus a parameterization of the observations which is essentially orthogonal to the best-fit parameters determined by the MACHO collaboration ${ }^{5}$.

${ }^{4}$ There remains a residual dependence of the Width on the typical MACHO mass, however, due to the duration dependence of the detection efficiencies.

5 This approach is similar to that of de Rujula et al. (1995) who showed that the duration dispersion could in principle be used to separate background events.
Table 1. Probability of various subsamples of the observed candidate microlensing events from the 5.7 year candidate events being drawn from an event duration distribution function (i.e. $\mathrm{d} \Gamma / \mathrm{d} t$ ) as predicted by the standard MW halo model. Here the columns indicate the number of candidate events $\left(N_{\mathrm{obs}}=13\right.$ for efficiency A, and $N_{\mathrm{obs}}=17$ for efficiency B), whether a delta- (DMF) or Gaussian- (GMF) MACHO mass function has been assumed, the numbers of the events which have been excluded from the sample by hand (correspondingly reducing the number of observed events from $N_{\mathrm{obs}}$ to $N_{\mathrm{obs}}^{*}$ ), the assumed average number $<N_{\mathrm{BG}}>^{*}$ of events due to non-halo background (BG) lens populations, as well as the aforementioned probabilities, computed either by subtracting a fixed number $\left\langle N_{\mathrm{BG}}>^{*}\right.$ from $N_{\mathrm{obs}}^{*}$ to obtain the number of halo events $N_{\mathrm{obs}}^{\text {halo }}$ to be drawn from the theoretical event duration distribution, or by finding the number $N_{\mathrm{BG}}^{*}$ of events to be subtracted from $N_{\mathrm{obs}}^{*}$, from a Poisson distribution with average $<N_{\mathrm{BG}}>^{*}$. In the case of non-integer $<N_{\mathrm{BG}}>^{*}$, this number is rounded up to the next integer, when fixed number background subtraction is performed. Note that the exclusion of event number 22 from the sample by hand is not assumed to reduce $<N_{\mathrm{BG}}>$ from 3.9 to 2.9 , as it is a supernova rather than microlensing due to a background lensing population.

\begin{tabular}{cccccc}
\hline \hline $\begin{array}{c}\text { Model/ } \\
\text { Sample }\end{array}$ & $\begin{array}{c}\text { mass } \\
\text { funct. }\end{array}$ & $\begin{array}{c}\text { events } \\
\text { excluded }\end{array}$ & $\begin{array}{c}\text { \# BG } \\
\text { events }\end{array}$ & $\begin{array}{c}\text { Prob. } \\
\text { (fixed) }\end{array}$ & $\begin{array}{c}\text { Prob. } \\
\text { (Poisson) }\end{array}$ \\
\hline S/13 & DMF & none & 3.0 & $6.9 \%$ & $8.9 \%$ \\
S/13 & GMF & none & 3.0 & $3.5 \%$ & $5.2 \%$ \\
S/13 & DMF & 5 & 2.0 & $6.9 \%$ & $9.1 \%$ \\
S/13 & GMF & 5 & 2.0 & $3.5 \%$ & $5.2 \%$ \\
S/13 & DMF & 5,14 & 1.0 & $8.1 \%$ & $8.7 \%$ \\
S/13 & GMF & 5,14 & 1.0 & $4.2 \%$ & $4.7 \%$ \\
S/17 & DMF & 22 & 3.9 & $6.4 \%$ & $7.7 \%$ \\
S/17 & GMF & 22 & 3.9 & $3.1 \%$ & $4.2 \%$ \\
S/17 & DMF & $5,9,14,22$ & 0.9 & $4.7 \%$ & $4.8 \%$ \\
S/17 & GMF & $5,9,14,22$ & 0.9 & $2.2 \%$ & $2.3 \%$ \\
\hline
\end{tabular}

In our Monte Carlo analysis we find the fraction of generated event samples that yield a Width which is smaller than that observed. To account for background (i.e. non-halo) lenses we employ the estimates for the expected number of background events $<N_{\mathrm{BG}}>$ found in Alcock et al. (2000b). We do not distinguish between the different background populations, but simply subtract a number $N_{\mathrm{BG}}^{*}$ from $N_{\mathrm{obs}}^{*}$ to obtain $N_{\mathrm{obs}}^{\text {halo }}$, the number of events which are expected to be due to MACHOs and are to be generated from the theoretical event duration distribution. We do this using two different methods: subtracting a fixed number $N_{\mathrm{BG}}^{*}=<N_{\mathrm{BG}}>^{*}$, and subtracting $N_{\mathrm{BG}}^{*}$ as drawn from a Poisson distribution with average $<N_{\mathrm{BG}}>^{* 6}$. We note here that while the background events could potentially account for the longest or shortest events in the sample, we nevertheless do not change the observed Width on subtracting background events, such that our estimated probabilities, in a conservative spirit, should generally reflect an overestimate.

The results of this procedure, in particular the fraction of simulations which have smaller Width than observed, are shown in Table 1 for a variety of different subsamples.

\footnotetext{
6 In some of our samples $<N_{\mathrm{BG}}>$ as given in Alcock et al. (2000b) is adjusted to $<N_{\mathrm{BG}}>^{*}$ to account for events which have been excluded from the sample by hand, cf. Table 1 .
} 
This fraction is generally between $5 \%$ and $10 \%$, such that the underlying S-DMF model serving as an explanation for the observations is ruled out at the 90 to $95 \%$ confidence level. Note that this conclusion does not vary strongly if the events which are suspected of being due to background populations are excluded by hand. This would not be the case if event number 22 , which has a duration of 297.8 days, was a genuine microlensing event.

The discrepancy between the observations and the theoretical expectations even worsens when the assumption of a delta function MACHO mass function (DMF) is dropped. To illustrate the effects of a MACHO mass function with finite width we take the number of MACHOs with mass within a logarithmic interval to be

$\frac{\mathrm{d} P}{\mathrm{~d} \ln \left(M / M^{*}\right)}=\frac{1}{\sqrt{2 \pi}} \frac{1}{\sigma} \exp \left\{-\frac{1}{2}\left(\frac{\ln \left(\frac{M}{M^{*}}\right)}{\sigma}\right)^{2}\right\}$,

with

$\sigma=\sqrt{\ln 2 / 2}$

such that MACHOs with either twice or one-half the average mass $<M>=\exp \left(\sigma^{2} / 2\right) M^{*}$ (which is assumed to take the best-fit value of the DMF) are a factor of two less common. These models are indicated in the table by GMF.

It may be noted that the probabilities are generally larger when background event subtraction is performed by employing Poisson statistics, rather than by subtracting a fixed number of events. This is due to the probabilities in the Poisson case being dominated by samples which have $N_{\mathrm{BG}}^{*}$ larger than $<N_{\mathrm{BG}}>^{*}$, and have correspondingly smaller $N_{\mathrm{obs}}^{\text {halo }}$, making a comparison of theoretical expectation and observed distribution more favorable. Nevertheless, such reasoning makes the implicit and nontrivial assumption that all background events have event durations falling within the range of those of a putative MACHO population.

One may ask, making this assumption about coincidence in event durations between different populations, by how much one has to increase the number of background events, in order to achieve a "decent" probability (say 20\%) of the observed distribution indeed being drawn from the distribution expected for the standard halo model. Figure 2 shows the probability for different samples, for the delta mass function (DMF) and that given by Eq. (5) (GMF), indicating that an increase of $N_{\mathrm{BG}}^{*}$ by about a factor of two would be necessary in order for this to be the case. This suggests, on statistical grounds, that the contribution of the background lens populations to the observed events has been underestimated, and consequently the MACHO halo mass fraction overestimated. Nevertheless, due to the small number of observed events, a significant contribution to the observed events by a population of MACHOs within a standard MW halo may not be ruled out. This is interesting in light of the recent detection of a population of old very cool white dwarfs which could comprise a few per-cent (Oppenheimer et al. 2001) of the MW dark halo, see Richer (2001) for a critical discussion of these observations.

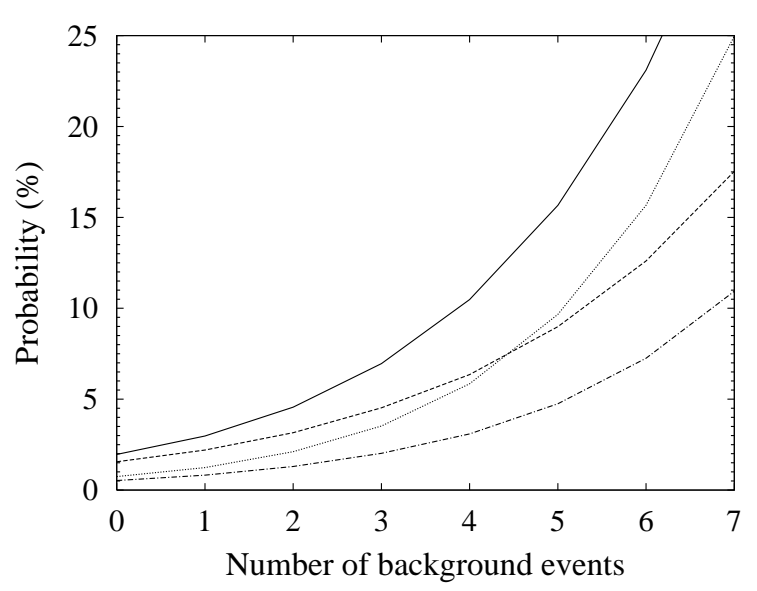

Fig. 2. Probability of obtaining an event duration distribution for a standard halo model as narrow as that observed in the 5.7 year sample as a function of the number of events assumed to be due to background population(s). Here the background events are implicitly assumed to neither contribute the longest nor shortest event, but to fall, by pure coincidence, within the range of the event durations of the putative MACHO population. Background subtraction is performed by subtracting a fixed (non-Poissonian) number of events from the number of observed candidate events. The lines show results for model/sample S/13 - DMF (solid), S/13 - GMF (dotted), S/17 - DMF (dashed), and S/17 - GMF (dashed-dotted), respectively, where in the latter two samples event number 22 has been excluded.

\section{Conclusions}

In this paper, we have shown that the most straightforward interpretation of the existing microlensing observations of stars within the LMC, assuming the majority of candidate events to be due to compact lenses within the MW halo, may actually be in conflict with the observational data itself. The MACHO collaboration uses a likelihood analysis to derive two "best-fit" parameters from their microlensing observations: the typical MACHO mass and MACHO halo fraction. A naive comparison of the theoretical event rate distribution produced by these "best-fits" assuming a standard halo model, with an ad-hoc Gaussian event rate distribution indicates that the spread in the durations of the observed events seems smaller than would be expected for a standard halo of compact dark matter. In order to quantify this suspicion we have formulated a statistic, the Width (essentially the spread in the observed event durations relative to the average event duration), that is virtually independent of the MACHO best-fit parameter values.

We have carried out Monte Carlo simulations to calculate the expected distribution of the Width, under the assumption of a standard halo MACHO population, utilizing two different background subtraction methods (fixed number and Poissonian), for both detection criteria, for delta function and finite width MACHO mass functions and excluded various sets of events suspected of being due to background populations. The probability of observing a value of the Width as small as observed falls, in all cases, below $10 \%$, with values often below 5\%. In other words the underlying model, in terms of the expected background event rate and a standard halo MACHO 
population, is excluded at between the $90 \%$ and $95 \%$ confidence level.

The discrepancy between the observational data and its halo dark matter interpretation may be alleviated if the number of events which are due to non-halo populations is significantly larger than expected, at the expense of correspondingly reducing the MACHO halo fraction. Such a reasoning also implicitly assumes the non-halo population to produce microlensing events with event durations being by chance very similar to those of the halo population. Possibly more natural would be an explanation in terms of "all background", though the nature of this background, and the reason for it producing such a narrow distribution in durations, remains to be established. Alternatively, the discrepancy may indicate that the standard halo model is a poor approximation to the actual MACHO distribution (Widrow \& Dubinski 1998). For instance, there could be a clump with small intrinsic velocity dispersion crossing the line of sight to the LMC, which, nevertheless, would be required to be surprisingly massive. Another possible resolution to the problem could be that the statistical correction to the event durations, due to blending, leads to a reduction in the spread of the durations i.e. that the underlying durations of some observed events are significantly longer than their estimated values. If this were the case, however, then conclusions based on these estimated timescales, not only the analysis in this paper, but also the derivation of exclusion limits on the halo fraction in massive black holes (Alcock et al. 2001a) due to the purported absence of long-duration events, would seem unreliable.

Further clarification of the nature of the lenses may hopefully result from an enlarged sample of microlensing candidates, as expected from the upcoming analysis of the MACHO 8 year data and EROS 5 year. Evidently a longer survey duration will increase the number of candidate events, reducing the ambiguity associated with Poisson statistics, as well as increasing the efficiency for detecting longer duration events, thus making the width of the distribution a significant discriminator between models. Nevertheless, a significant reduction of Poisson ambiguity may only result by an increase of the number of events by an order of magnitude, such as anticipated in the next generation SUPERMACHO survey (Stubbs 1998). This later survey is expected to also reduce uncertainties in event duration corrections due to blending as it is planned to employ the technique of difference image analysis.
Acknowledgements. We are grateful to David Bennett, Alain Milsztajn and Esteban Roulet for useful comments. AMG was supported by PPARC and the Swedish Research Council, and acknowledges the use of the starlink computing facilities at QMW.

\section{References}

Alcock, C., Allsman, R. A., Axelrod, T. S., et al. 1996, ApJ, 461, 84 Alcock, C., Allsman, R. A., Alves, D. R., et al. 1997, ApJ, 486, 697

Alcock, C., Allsman, R. A., Alves, D. R., et al. 2000a, ApJ, 541, 270

Alcock, C., Allsman, R. A., Alves, D. R., et al. 2000b, ApJ, 542, 281

Alcock, C., Allsman, R. A., Alves, D. R., et al. 2001a, ApJ, 550, 169

Alcock, C., Allsman, R. A., Alves, D. R., et al. 2001b, Nature, 414, 617

Alcock, C., Allsman, R. A., Alves, D. R., et al. 2001c, ApJ, 552, 259

Alcock, C., Allsman, R. A., Alves, D. R., et al. 2001d, ApJS, 136, 439

Aubourg, E., Palanque-Delabrouille, N., Salati, P., Spiro, M., \& Taillet, R. 1999, A\&A, 347, 850

Bennett, D. P., Alcock, C., Allsman, R. A., et al. 1996, Nucl. Phys. B. Proc. Suppl, 51, 131

Bennett, D. P. 1998, Phys. Rep., 307, 97

de Rujula, A., Giudice, G. F., Mollerach, S., \& Roulet, E. 1995, MNRAS, 275, 545

Evans, N. W., \& Kerins, E. J. 2000, ApJ, 529, 917

Gyuk, G., Dalal, N., \& Griest, K. 2000, ApJ, 535, 90

Gyuk, G., \& Gates, E. 2001, ApJ, 547, 786

Honma, M. 1999, ApJ, 511, L29

Lasserre, T., Afonso, C., Albert, J. N., et al. 2000, A\&A, 355, 39

Milsztajn, A., \& Lasserre, T. 2001, Nucl. Phys. B (Proc. Suppl.), 91, 413

Milsztajn, A. 2002, Space Sci. Rev., 100, 103

Oppenheimer, B. R., Hambly, N. C., Digby, A. P., Hodgkin, S. T., \& Saumon, D. 2001, Science, 292, 698

Paczyński, B. 1986, ApJ, 428, L5

Richer, H. B. 2001, in the Proc. of The Dark Universe: Matter, Energy and Gravity [astro-ph/0107079]

Salati, P., Taillet, R., Auborg, E., Palanque-Delabrouille, N., \& Spiro, M. 1999, A\&A, 350, L57

Stubbs, C. 1998, in The Third Stromlo Symp., The Galactic Halo, ed. B. K Gibson, T. S. Axelrod, \& M. E. Putman, ASP Conf. Ser., 165,503

Widrow, L. M., \& Dubinski, J. 1998, ApJ, 504, 12

Wu, X.-P. 1994, ApJ, 435, 66

Zhao, H. 1999, COSMO-98, Second International Workshop on Particle Physics and the Early Universe, ed. D. O. Caldwell (American Institute of Physics, Woodbury, N.Y.), AIP Conf. Proc., 478, 305 